\title{
Local Search with Noisy Strategy for Minimum Vertex Cover in Massive Graphs
}

\author{
Zongjie Ma ${ }^{1 \star}$, Yi Fan ${ }^{1}$, Kaile $\mathrm{Su}^{1}$, Chengqian $\mathrm{Li}^{2}$, and Abdul Sattar ${ }^{1}$ \\ 1 Institute for Integrated and Intelligent Systems, Griffith University, Brisbane, \\ Australia \\ 2 Department of Computer Science, Sun Yat-sen University, China \\ zongjie.ma@griffithuni.edu.au
}

\begin{abstract}
Finding minimum vertex covers (MinVC) for simple undirected graphs is a well-known NP-hard problem. In the literature there have been many heuristics for obtaining good vertex covers. However, most of them focus on solving this problem in relatively small graphs. Recently, a local search solver called FastVC is designed to solve the MinVC problem on real-world massive graphs. Since the traditional bestpicking heuristic was believed to be of high complexity, FastVC replaces it with an approximate best-picking strategy. However, since best-picking has been proved to be powerful for a wide range of problems, abandoning it may be a great sacrifice. In this paper we have developed a local search MinVC solver which utilizes best-picking with noise to remove vertices. Experiments conducted on a broad range of real-world massive graphs show that our proposed method finds better vertex covers than state-of-the-art local search algorithms on many graphs.
\end{abstract}

Keywords: Minimum Vertex Cover, Heuristic Search, Massive Graphs, Combinatorial Optimization, Social Networks

\section{Introduction}

The rapid growth of the Internet, widespread deployment of sensors and other fields produced huge quantity of massive data sets, which has generated a series of computational challenges to existing algorithms. Hence, new algorithms need to be designed to deal with these data sets. Many of these data can be modeled as graphs, and the interest in real-world massive graphs, also known as complex networks [21], is growing significantly over recent decades.

The Minimum Vertex Cover (MinVC) problem is a fundamental NP-hard problem in computer science. Given a simple undirected graph $G$, a vertex cover $S$ is a subset of vertices s.t. every edge in $G$ has at least one endpoint in $S$. The objective of MinVC is to find a vertex cover of the minimum size. MinVC is one of the well-known optimization problems with many real-world applications, such as network security, scheduling, VLSI design and industrial machine assignment

\footnotetext{
* Corresponding author
} 
$[5,16]$. Also the MinVC problem is closely related to the Maximum Independent Set (MIS) and Maximum Clique (MC) problems, in that algorithms for MinVC can be directly applied to solve the MIS and MC problems. The applications of these three problems involve computer version, information retrieval, signal transmission, aligning DNA and protein sequences [15, 10,11], etc.

\subsection{Previous Heuristics and Motivations}

Due to the great importance to many real-world applications, a large number of algorithms for solving MinVC (MIS, MC) have been proposed during the past decades. Practical algorithms for them can be roughly grouped into two categories, i.e., exact algorithms and heuristic algorithms. The exact ones, mainly based on the general branch-and-bound framework [14,20], confirm the optimality of the solutions they find. However, for large and hard instances, exact methods may become ineffective and fail to return a solution within reasonable time.

On the other hand, heuristic methods are able to find near-optimal solutions within reasonable time for large and hard instances. Local search is a popular strategy among the heuristic approaches, such as $[5,16]$ for MinVC, $[1,2]$ for MIS, and [13] for MC.

The evaluation of existing local search approaches for MinVC are mainly based on standard benchmarks from academic community, such as the DIMACS [12] and BHOSLIB ${ }^{3}$ benchmarks [5,16]. In order to improve the performance on these benchmarks, a number of heuristics combined with local search for MinVC have been proposed in the literature recently. COVER [16] introduces edge weighting to MinVC, and is an iterative best improvement approach through updating edge weights at each step to guide a local search. EWLS [4] also exploits the edge weighting strategy, but it only updates the edge weights when being stuck in local optima. EWCC [6] introduces the configuration checking $(\mathrm{CC})$ heuristic into EWLS, and CC is a strategy for handling the cycling problem in local search. Especially, NuMVC [5] introduces two strategies, named two-stage exchange and edge weighting with forgetting, and makes a significant improvement in MinVC solving. Since the benchmarks graphs used by these previous algorithms are not large (usually with less than five thousand vertices), the impact of the complexity of heuristics on the performance is not significant.

In this work, we focus on studying the local search for MinVC in massive real world graphs. Many of these real world graphs have millions of vertices and dozens of millions of edges [17]. The complexity of most previous heuristics is not sufficiently small, and they suffer from these massive graphs with millions of vertices. Thus massive graphs call for new heuristics and algorithms. Recently, an algorithm called FastVC [3] takes a first step towards solving the MinVC problem for real-world massive graphs. FastVC outperforms other existing local search algorithms on finding vertex covers in massive graphs. It is designed by withdrawing or modifying some techniques with high computational cost in

\footnotetext{
${ }^{3}$ http://www.nlsde.buaa.edu.cn/ kexu/benchmarks/graph-benchmarks.htm
} 
NuMVC [5]. Specifically, FastVC replaces the best-picking heuristic in NuMVC with a low-complexity heuristic named Best from Multiple Selection (BMS). BMS approximates the best-picking heuristic very well.

In local search phase, FastVC abandons the best-picking heuristic in NuMVC. However, this best-picking heuristic guides the search towards very promising areas with a suitable criterion, and is thus widely used in local search algorithms. Besides, when finding a $k$-vertex cover, FastVC exploits traditional best-picking strategy to remove a vertex with minimum loss to generate a $(k-1)$-candidate solution. However, our experiments show that this process happens very frequently, which can be time-consuming due to the $O(|V|)$ complexity.

\subsection{Contribution and Paper Organization}

In this work, we propose a new algorithm called NoiseVC, which is dedicated to solve the MinVC problem in massive graphs. Instead of abandoning the best-picking heuristic, we exploited an efficient data structure named min-heap for best-picking in this work. The procedure of min-heap [8] runs in $O(|\lg V|)$ to maintain its property for best-picking, while the complexity of traditional best-picking heuristic in NuMVC is believed to be $O(|V|)$ which is very timeconsuming for massive graphs.

Since best-picking can easily be trapped by local minima, we design a noisy strategy to help it escape from local minima. Given a candidate vertex set $C$, with probability $p$, remove a vertex with the minimum loss, breaking ties in favor of the oldest one; with probability $1-p$, remove a vertex randomly.

Besides, when finding a $k$-vertex cover, min-heap is also used to remove a vertex with the minimum loss to generate a $(k-1)$-candidate solution, which lowers the complexity and helps to save time in updating the best solution.

We conduct experiments to compare NoiseVC with FastVC on a wide range of real-world massive graphs. Experimental results show that for all the 12 classes of instances in this benchmark, NoiseVC significantly outperforms FastVC on solution quality for 8 classes. More specifically, over the 136 graphs we tested, NoiseVC finds smaller vertex cover on 23 graphs.

Using the 23 graphs above, we tested FastVC with a cutoff of 100,000 seconds. Even within such a large cutoff the solutions obtained by FastVC are still worse than those obtained by NoiseVC within 1000 seconds. This means that NoiseVC is at least 100 times as efficient as FastVC on these graphs. It rarely happens in literature to find a better solution. The existing MinVC algorithms often obtain the same quality solutions, and concern on comparing the success rate of finding a solution of such a quality.

The rest of paper is organized as follows. Sections 2 gives some necessary definitions and notations, and shows the framework of the local search for MinVC problem . Then, we describe our new algorithm for MinVC on massive graphs in section 3 . In section 4 , we carry out extensive experiments to evaluate NoiseVC. Finally, we conclude our work in section 5 . 


\section{Preliminaries}

\subsection{Definitions and Notation}

Given an undirected graph $G=(V, E)$, where $V=\left\{v_{1}, v_{2}, \ldots v_{n}\right\}$ is a vertex set and $E \subseteq V \times V$ is an edge set. Each edge is a 2-element subset of $V$. For an edge $e=\{u, v\}$, the vertices $u$ and $v$ are called the endpoints of edge $e$. Two vertices, such as $u$ and $v$, are neighbors if and only if there exists an edge between them. The neighborhood of $v$ is defined as $N(v)=\{u \in V \mid\{u, v\} \in E\}$. The degree of $v$ is defined as $\operatorname{deg}(v)=|N(v)|$, which is equal to the number of its neighbors. An edge $e \in E$ is covered by a vertex set $S \subseteq V$ if at least one endpoint of $e$ is in $S$; otherwise, $e$ is uncovered by $S$.

For a graph $G=(V, E)$, a vertex cover of $G$ is a subset of $V$ which contains one or two endpoints of each edge in $E$. The complementary graph of $G$ is denoted as $\bar{G}=(V, \bar{E})$, where $\bar{E}=\{(u, v) \mid(u, v) \notin E\}$. Then for a subset $S \subseteq V$, there are three equivalent statements [22]: $S$ is a vertex cover of $G, V \backslash S$ is an independent set of $G$ and $V \backslash S$ is a clique of $\bar{G}$.

\subsection{Local Search for MinVC}

We use $C$ to denote the current candidate solution, which is a set of vertices selected for covering. Algorithm 1 shows a general framework of local search for MinVC.

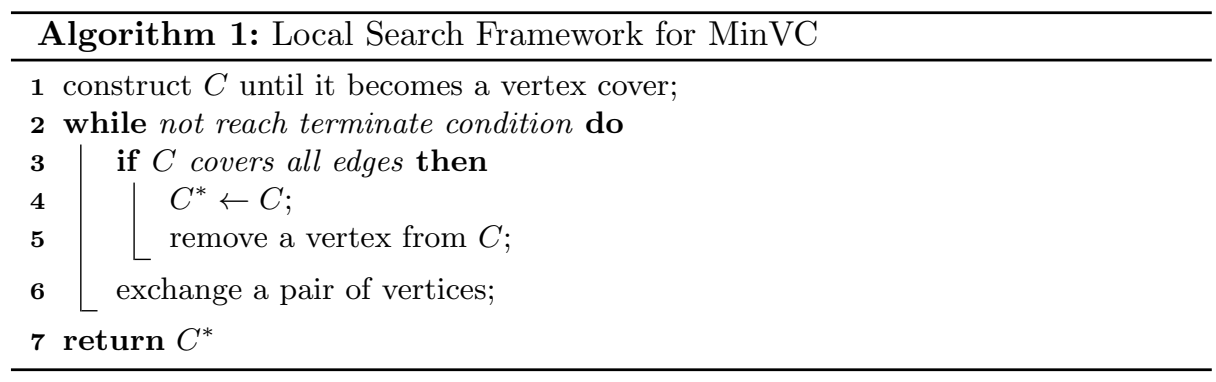

There are two phases in Algorithm 1: a construction stage (Line 1) and a local search phase (Lines 2 to 6 ). A vertex cover is constructed at the first phase, and such a vertex cover is also called the starting vertex cover throughout this paper. In the local search phase, whenever the algorithm finds out a $k$-sized cover (Line 3), one vertex is removed from $C$ (Line 5) and continues to search for a $(k-1)$-sized cover, until some termination condition is reached (Line 2).

The move to a neighboring candidate solution consists of an exchange of two vertices (Line 6): a vertex $u \in C$ is removed from $C$ and a vertex $v \notin C$ is added into $C$. This step is also called an exchanging step. Thus the local search moves step by step in the search space to find a smaller vertex cover. After the algorithm terminates, it outputs the smallest vertex cover that has been found. 
For a vertex $v \in C$, the loss of $v$, denoted as $\operatorname{loss}(v)$, is defined as the number of covered edges that will become uncovered by removing $v$ from $C$. For a vertex $v \notin C$, the gain of $v$, denoted as gain $(v)$, is defined as the number of uncovered edges that will become covered by adding $v$ into $C$. Both loss and gain stand for scoring properties of vertices. In any step, a vertex $v$ has two possible states: inside $C$ and outside $C$. We use $a g e(v)$ to denote the number of steps since its state was last changed.

\section{$3 \quad$ NoiseVC for MinVC in Massive Graphs}

\subsection{The Top-Level Algorithm of NoiseVC}

We describe the NoiseVC algorithm on a top level in this subsection and the details of the proposed functions will be given and analyzed in the next subsection.

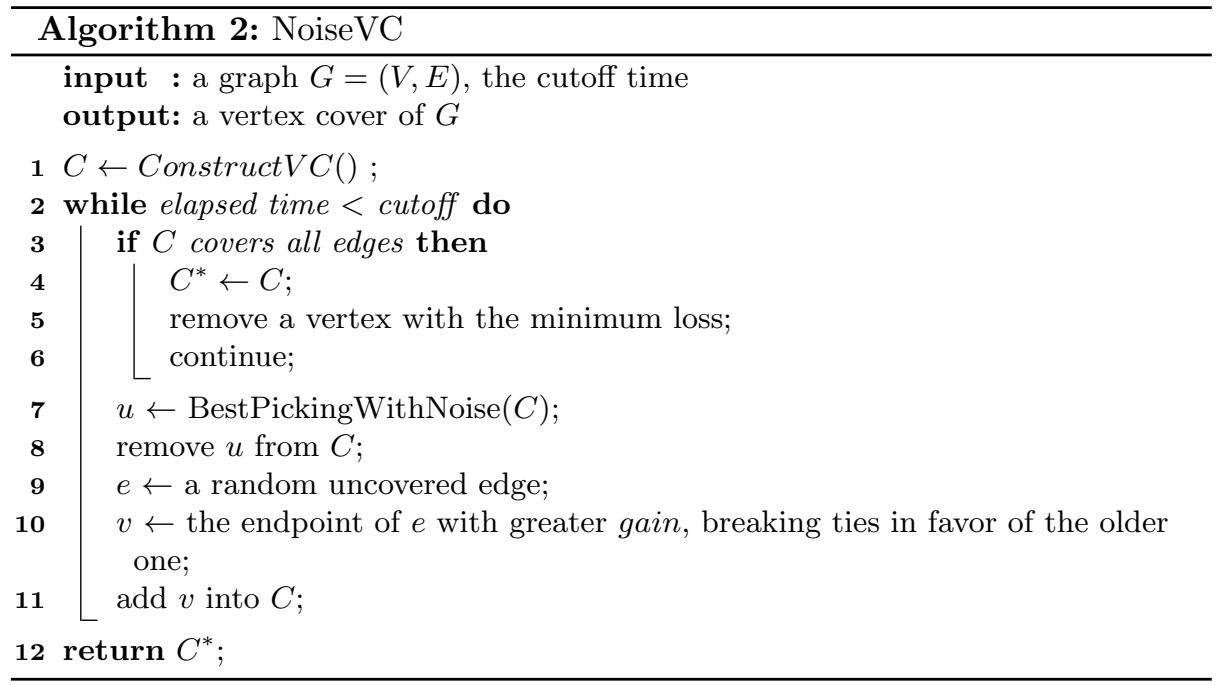

NoiseVC outlined in Algorithm 2 adopts the local search framework for MinVC in Algorithm 1. In the construction phase (Line 1), a starting vertex cover is constructed by a function named Construct $V C()$ which is introduced in FastVC. In the local search phase (Lines 2 to 11), whenever NoiseVC obtains a vertex cover, a vertex with minimum loss is removed based on the min-heap data structure. In the exchanging step (Lines 7 to 11), NoiseVC first chooses a vertex in $C$ to remove, which is accomplished by the BestPickingWithNoise() function. Then the algorithm selects an uncovered edge randomly and chooses the endpoint of this edge with greater gain (breaking ties in favor of the older one) to add it into $C$. It should be noted that along with removing or adding a vertex, the algorithm will update the loss and gain values of the vertex and its neighbors. 


\subsection{Min-heap for Best-picking in Massive Graphs}

Many of these real world graphs have millions of vertices and dozens of millions of edges. The traditional best-picking heuristic, which aims at choosing the best element according to some criterion, is very time-consuming for these massive data sets. Therefore, in FastVC best-picking heuristic is replaced by BMS, which approximates best-picking very well. BMS works as follows: Choose $k$ vertex randomly with replacement from $C$, and then return a vertex with the minimum loss, breaking ties in favor of the oldest one. Since $k$ is set to 50 in FastVC, BMS chooses a vertex whose loss value is among the best $10 \%$ in $C$ with probability of $99.48 \%$. However, the best $10 \%$ can be a very large number in massive graphs.

Instead of abandoning best-picking heuristic, we exploit min-heap [8] for bestpicking in this work. The vertices in $C$ are used to build a min-heap according to their loss value, which is used for choosing the vertex with the minimum loss. The vertex with the minimum loss is returned by heap-minimum $(C)$ in our min-heap. Throughout this paper, we use heap-minimum $(C)$ to denote the result of best-picking by min-heap. To the best of our knowledge, it is the first time that a heap is used for best-picking in MinVC solving.

Complexity Analysis Along with remove or add a vertex $v$, we have to update the loss value of its neighbors. Therefore the worst complexity of our best-picking is $O\left(d_{\max }|\lg V|\right)$, where $d_{\max }$ is the maximum degree in a graph. Since most massive real world graphs are sparse graphs $[9,7]$, the value of $d_{\max }$ is usually not large. On the other hand, the complexity of traditional best-picking heuristic in NuMVC is $O(|V|)$ which is very time-consuming for massive graphs.

\subsection{Best-picking with noisy strategy}

During the exchanging step, best-picking can easily be trapped by local minima. In this work, we introduce noise to help it escape from local minima. When choosing a vertex to remove, BestPickingWithNoise() is shown in Algorithm 3.

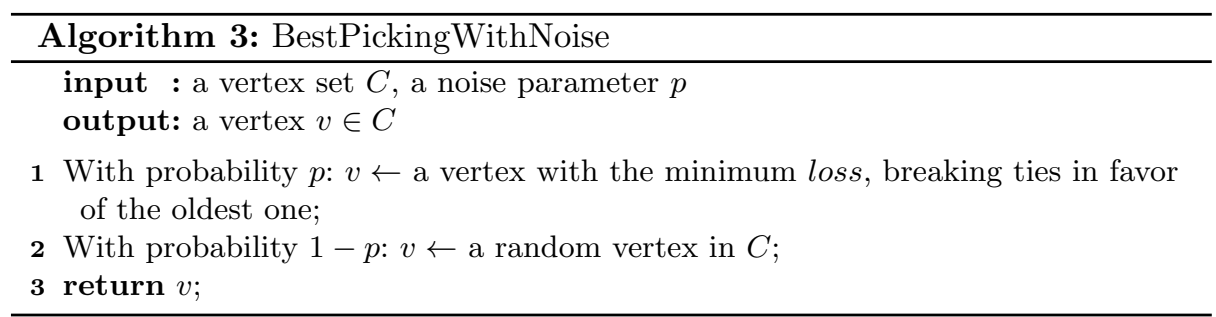

Throughout this paper, the parameter $p$ is fixed to 0.4 in advance for all the experiments. This means that the parameter $p$ in this study is instanceindependent. Different $p$ values are only used to test parameter sensitivity. 
There are two modes in Algorithm 3: the greedy mode (Line 1) and the random mode (Line 2). BestPickingWithNoise() switches between the two modes at a certain probability. In the greedy mode, a vertex with minimum loss is returned by heap-minimum $(C)$. In the random mode, a vertex is selected randomly to avoid local optima.

Relationship with Other Heuristics The proposed best-picking with noisy strategy is a new kind of random walk, which is an efficient and effective method with very low time complexity to improve local search. The existing random walk strategy focused on choosing a variable (vertex) from a random unsatisfied constraint (unsatisfied clause or uncovered edge). Our random walk strategy considers all satisfied constraints, and it chooses a vertex from $\mathrm{C}$ where all the incident edges are covered.

\section{Experimental Results and Analysis}

This section compares NoiseVC with FastVC using real-world massive graphs, since FastVC outperforms other existing local search algorithms on finding vertex covers in massive graphs.

\subsection{Benchmark}

To verify the effectiveness of our method, we conducted comparative experiments on a broad range of real-world massive graphs. All 139 instances are downloaded $^{4}$, which were originally online ${ }^{5}$. We excluded three extremely large ones, since they are out of memory for both solvers here. Many of these realworld massive graphs contain millions of vertices and dozens of millions of edges. Recently, some of these graphs are used to test parallel algorithms for Maximum Clique [19] and Coloring problems [18].

The benchmark used in our experiments can be divided into 12 classes: biological networks, collaboration networks, Facebook networks, interaction networks, infrastructure networks, Amazon recommend networks, retweet networks, scientific computation networks, social networks, technological networks, web lint networks, and temporal reachability networks.

\section{$4.2 \quad$ Setup}

NoiseVC was implemented in $\mathrm{C}++^{6}$, and FastVC was also implemented in $\mathrm{C}++^{7}$. Both solvers were compiled by $\mathrm{g}++4.6 .3$ with the option $-\mathrm{O} 3$. The

\footnotetext{
${ }^{4}$ http://lcs.ios.ac.cn/ caisw/Resource/realworld\%20graphs. tar.gz

${ }^{5}$ http://www.graphrepository.com/networks.php

${ }^{6}$ https://github.com/math6068/NoiseVC

${ }^{7}$ http://lcs.ios.ac.cn/ caisw/Code/FastVCv2015.11.zip
} 
experiments were conducted on a cluster equipped with a number of Intel(R) Xeon(R) central processing units (CPUs) X5650 @2.67GHz with 8GB RAM, running Red Hat Santiago OS. In our experiments, the probability parameter $p$ is fixed to be 0.4. For FastVC, we adopt the parameter setting reported in [3]. Both solvers are run 10 times on each instance with a time limit of 1000 seconds if not mentioned explicitly. For each solver on each instance, we report the minimum size (" $C_{\text {min }}$ ") and averaged size (" $C_{\text {avg }}$ ") of vertex covers found by the solver. To clarify the comparisons, we report the difference (" $\Delta$ ") between the minimum sizes of vertex cover found by NoiseVC and FastVC. A positive (negative) means NoiseVC (FastVC) finds a smaller vertex cover.

\subsection{Experimental Results}

The main experimental results are shown in Table 1. We tested all the 139 instances, and the results on graphs where NoiseVC and FastVC return precisely return solutions with both the same $C_{\min }$ and $C_{a v g}$ are not reported in Table 1 .

According to the results in Table 1, we observe that:

1. Out of 44 graphs, NoiseVC finds better and worse vertex covers than FastVC in 23 and 8 graphs, respectively.

2. NoiseVC finds the same minimum vertex cover as FastVC in 13 graphs, among which NoiseVC obtains smaller average size of vertex cover for 10 graphs (bold value in Table 1).

3. NoiseVC outperforms FastVC for 8 classes of instances listed in Table 1, except for technological networks and web link networks.

Table 2 shows the performances on those instances where two solvers return both the same $C_{\min }$ and $C_{\text {avg }}$ values. We also present the averaged number of steps to locate a solution, and the number of steps executed in each millisecond. The time columns show that NoiseVC outperforms FastVC on 24 instances, while FastVC is faster than NoiseVC on 10 instances. The last two columns reveal that the complexity per step in NoiseVC is significantly lower than that in FastVC.

Speed Improvements Over a half of the 23 graphs where we found smaller covers, NoiseVC makes a substantially large progress. Now we show how great the progress is. We enlarged the cutoff to be 100 times as large as before (i.e., 100,000s), and tested FastVC over such graphs. The results are shown in Table 3 . Also we present the respective results of NoiseVC within $\mathbf{1 , 0 0 0}$ seconds in this table.

As is shown in Table 3, even within such a large cutoff, FastVC does not get the same solution quality as NoiseVC does with a cutoff of 1,000 seconds for any of these 12 graphs. That is, our solver is at least 100 times as efficient as FastVC on these graphs.

Parameter Testing Table 4 shows the results of NoiseVC with different parameter $p$ ranging from 0.1 to 0.9 . We make a comparison with FastVC on average $\Delta$ over 136 instances. NoiseVC outperforms FastVC significantly on a wide range of parameter settings, from 0.1 to 0.6 . 
Table 1. Experimental results on real-world massive graphs. A positive $\Delta$ means NoiseVC finds a smaller vertex cover, while a negative $\Delta$ means FastVC finds a smaller vertex cover. For $\Delta \neq 0$, we bold the smaller value of minimum size $\left(C_{\text {min }}\right)$ between the two algorithms, and for $\Delta=0$, we bold the smaller value of average size $\left(C_{a v g}\right)$

\begin{tabular}{|c|c|c|c|c|c|}
\hline Graph & $|V|$ & $|E|$ & $\begin{array}{l}\text { FastVC } \\
C_{\min }\left(C_{a v g}\right)\end{array}$ & $\begin{array}{l}\text { NoiseVC } \\
C_{\min }\left(C_{a v g}\right)\end{array}$ & $\Delta$ \\
\hline socfb-A-anon & 3097165 & 23667394 & $\mathbf{3 7 5 2 3 1}(375232.8)$ & $375233(375233)$ & -2 \\
\hline socfb-B-anon & 2937612 & 20959854 & $303048(303048.8)$ & $303049(303049)$ & -1 \\
\hline socfb-Berkeley 13 & 22900 & 852419 & $17210(17212.8)$ & $17210(\mathbf{1 7 2 1 2 . 1})$ & 0 \\
\hline socfb-CMU & 6621 & 249959 & $4986(4986.5)$ & $4986(\mathbf{4 9 8 6})$ & 0 \\
\hline socfb-Duke14 & 9885 & 506437 & $7683(7683.1)$ & $7683(\mathbf{7 6 8 3})$ & 0 \\
\hline socfb-Indiana & 29732 & 1305757 & $23315(23317.3)$ & $\mathbf{2 3 3 1 4}(23316.3)$ & 1 \\
\hline socfb-OR & 63392 & 816886 & $36548(36549.2)$ & $\mathbf{3 6 5 4 7}(36547.8)$ & 1 \\
\hline socfb-Penn94 & 41536 & 1362220 & $31162(31164.8)$ & $\mathbf{3 1 1 6 1 ( 3 1 1 6 4 )}$ & 1 \\
\hline socfb-Stanford3 & 11586 & 568309 & $8518(8518)$ & $\mathbf{8 5 1 7}(8517.6)$ & 1 \\
\hline socfb-Texas 84 & 36364 & 1590651 & $28167(28171.4)$ & $\mathbf{2 8 1 6 6 ( 2 8 1 7 0 . 3 )}$ & 1 \\
\hline socfb-UCLA & 20453 & 747604 & $15223(15224.3)$ & $15222(15223.8)$ & 1 \\
\hline socfb-UConn & 17206 & 604867 & $13230(13231.6)$ & $13230(\mathbf{1 3 2 3 1 . 2})$ & 0 \\
\hline socfb-UCSB37 & 14917 & 482215 & $11261(11263.1)$ & $11261(\mathbf{1 1 2 6 1 . 1})$ & 0 \\
\hline socfb-UF & 35111 & 1465654 & $27306(27309.1)$ & $27305(27308.3)$ & 1 \\
\hline socfb-UIllinois & 30795 & 1264421 & $24091(\mathbf{2 4 0 9 2 . 6})$ & $24091(24093.60)$ & 0 \\
\hline socfb-Wisconsin 87 & 23831 & 835946 & $18383(18385.1)$ & $18383(\mathbf{1 8 3 8 3 . 8})$ & 0 \\
\hline ia-infect-dublin & 410 & 2765 & $293(293.5)$ & $293(293)$ & 0 \\
\hline inf-roadNet-CA & 1957027 & 2760388 & $1001273(1001310.9)$ & $1001269(1001302)$ & 4 \\
\hline inf-roadNet-PA & 1087562 & 1541514 & $555220(555242.8)$ & $555191(555226.4)$ & 29 \\
\hline rec-amazon & 91813 & 125704 & $47606(47606)$ & $\mathbf{4 7 6 0 5 ( 4 7 6 0 5 . 5 )}$ & 1 \\
\hline rt-retweet-crawl & 1112702 & 2278852 & $81048(81048)$ & $\mathbf{8 1 0 4 6 ( 8 1 0 4 6 . 9 )}$ & 2 \\
\hline sc-ldoor & 952203 & 20770807 & $\mathbf{8 5 6 7 5 5 ( 8 5 6 7 5 7 . 4 )}$ & $856756(856758.2)$ & -1 \\
\hline sc-nasasrb & 54870 & 1311227 & $51244(51247.4)$ & $\mathbf{5 1 2 4 2}(51245.2)$ & 2 \\
\hline sc-pkustk11 & 87804 & 2565054 & $83911(83912.5)$ & 83911 (83911.8) & 0 \\
\hline sc-pkustk13 & 94893 & 3260967 & $\mathbf{8 9 2 1 7}(89220.6)$ & $89241(89248.5)$ & -24 \\
\hline sc-pwtk & 217891 & 5653221 & $207716(207719.9)$ & $207707(207715.2)$ & 9 \\
\hline sc-shipsec1 & 140385 & 1707759 & $117318(117338.4)$ & $117246(117273.6)$ & 72 \\
\hline sc-shipsec5 & 179104 & 2200076 & $147140(147175)$ & $147115(147142.8)$ & 25 \\
\hline soc-buzznet & 101163 & 2763066 & $30625(30625)$ & $\mathbf{3 0 6 1 8 ( 3 0 6 2 2 . 3 )}$ & 7 \\
\hline soc-delicious & 536108 & 1365961 & $85686(85696.4)$ & $85527(85596.9)$ & 159 \\
\hline soc-digg & 770799 & 5907132 & $103244(103245.3)$ & $103244(\mathbf{1 0 3 2 4 5})$ & 0 \\
\hline soc-flickr & 513969 & 3190452 & $153272(153272)$ & $153271(153272)$ & 1 \\
\hline soc-FourSquare & 639014 & 3214986 & $90109(90109.3)$ & $90108(90109.3)$ & 1 \\
\hline soc-gowalla & 196591 & 950327 & $84222(84222.3)$ & $84222(\mathbf{8 4 2 2 2 . 2})$ & 0 \\
\hline soc-livejournal & 4033137 & 27933062 & $1869045(1869053.7)$ & $1869036(1869048.2)$ & 9 \\
\hline soc-pokec & 1632803 & 22301964 & $843422(843434.8)$ & $843426(843433.6)$ & -4 \\
\hline tech-as-skitter & 1694616 & 11094209 & $\mathbf{5 2 7 1 8 5 ( 5 2 7 1 9 6 )}$ & $527253(527274.7)$ & -68 \\
\hline tech-RL-caida & 190914 & 607610 & $74930(74938.9)$ & $\mathbf{7 4 8 6 3}(74883.5)$ & 67 \\
\hline scc_infect-dublin & 10972 & 175573 & $9104(9104)$ & 9103(9103) & 1 \\
\hline web-arabic-2005 & 163598 & 1747269 & $\mathbf{1 1 4 4 2 6 ( 1 1 4 4 2 7 . 2 )}$ & $114427(114428.2)$ & -1 \\
\hline web-BerkStan & 12305 & 19500 & $\mathbf{5 3 8 4}(5384)$ & $5385(5385)$ & -1 \\
\hline web-it-2004 & 509338 & 7178413 & $414671(\mathbf{4 1 4 6 7 6 . 3})$ & $414671(414676.8)$ & 0 \\
\hline web-spam & 4767 & 37375 & $2298(2298)$ & $2297(2297)$ & 1 \\
\hline web-wikipedia2009 & 1864433 & 4507315 & $648317(\mathbf{6 4 8 3 2 1 . 7})$ & $648317(648323.5)$ & 0 \\
\hline
\end{tabular}

\section{Conclusions}

In this work, we propose a new algorithm named NoiseVC for MinVC in massive real-world graphs. A heap is exploited for best-picking in the local search phase, and a new noisy strategy is combined with best-picking to avoid local minima. The experimental results indicate that NoiseVC significantly outperforms FastVC on finding smaller vertex cover in most of the massive graphs of the benchmarks. In the future, we would like to design heap-based best-picking heuristic for other combinatorial optimization problems. 
Table 2. Comparative performances on instances where both solvers return the same $C_{\min }$ and $C_{a v g}$ values. We bold the better value between two solvers

\begin{tabular}{|c|c|c|c|c|}
\hline \multirow{2}{*}{ Graph } & & & & \\
\hline & FastVC & NoiseVC & FastVC & NoiseVC \\
\hline bio-celegans & $\overline{c<0.01}$ & $\overline{c<0.01}$ & 9992 & 19999 \\
\hline bio-diseasome & $<0.01$ & $<0.01$ & 794 & 2351 \\
\hline bio-dmela & $<0.01$ & 0.012 & 929 & 1515 \\
\hline bio-yeast & $<0.01$ & $<0.01$ & 903 & 2766 \\
\hline ca-AstroPh & 0.038 & 0.035 & 683 & 735 \\
\hline ca-citeseer & 1.503 & 0.562 & 334 & 811 \\
\hline ca-coauthors-dblp & 14.344 & 22.297 & 258 & 135 \\
\hline ca-CondMat & 0.030 & 0.021 & 678 & 1090 \\
\hline ca-CSphd & $<0.01$ & $<0.01$ & 919 & 4135 \\
\hline ca-dblp-2010 & 2.197 & 0.749 & 381 & 911 \\
\hline ca-dblp-2012 & 5.625 & 1.799 & 316 & 809 \\
\hline ca-Erdos992 & $<0.01$ & $<0.01$ & 1091 & 2687 \\
\hline ca-GrQc & $<0.01$ & $<0.01$ & 701 & 1575 \\
\hline ca-HepPh & 0.02 & 0.02 & 650 & 580 \\
\hline ca-hollywood-2009 & 25.814 & 50.033 & 220 & 104 \\
\hline ca-MathSciNet & 5.296 & 4.159 & 376 & 689 \\
\hline ca-netscience & $<0.01$ & $<0.01$ & 759 & 2520 \\
\hline ia-email-EU & $<0.01$ & $<0.01$ & 940 & 1006 \\
\hline ia-email-univ & $<0.01$ & $<0.01$ & 942 & 1562 \\
\hline ia-enron-large & 0.072 & 0.054 & 585 & 1150 \\
\hline ia-fb-messages & $<0.01$ & $<0.01$ & 1108 & 1649 \\
\hline ia-infect-hyper & $<0.01$ & $<0.01$ & 1041 & 653 \\
\hline ia-reality & $<0.01$ & $<0.01$ & 1153 & 1368 \\
\hline ia-wiki-Talk & 0.159 & 0.112 & 601 & 913 \\
\hline inf-power & 0.013 & $<0.01$ & 850 & 2889 \\
\hline rt-retweet & $<0.01$ & $<0.01$ & 962 & 4351 \\
\hline rt-twitter-copen & $<0.01$ & $<0.01$ & 863 & 3312 \\
\hline sc-msdoor & 18.180 & 37.031 & 312 & 143 \\
\hline socfb-MIT & 72.995 & 6.442 & 952 & 386 \\
\hline soc-BlogCatalog & 0.323 & 0.679 & 541 & 228 \\
\hline soc-brightkite & 0.281 & 0.219 & 648 & 1106 \\
\hline soc-dolphins & $<0.01$ & $<0.01$ & 1036 & 2427 \\
\hline soc-douban & 0.012 & 0.014 & 964 & 1002 \\
\hline soc-epinions & 0.231 & 0.176 & 619 & 1418 \\
\hline soc-flixter & 3.398 & 1.924 & 438 & 430 \\
\hline soc-lastfm & 1.637 & 1.604 & 617 & 493 \\
\hline soc-LiveMocha & 26.357 & 21.853 & 483 & 310 \\
\hline soc-slashdot & 0.287 & 0.301 & 547 & 818 \\
\hline soc-twitter-follows & 0.037 & 0.044 & 1004 & 211 \\
\hline soc-wiki-Vote & $<0.01$ & $<0.01$ & 951 & 2380 \\
\hline soc-youtube & 9.200 & 7.350 & 318 & 644 \\
\hline soc-youtube-snap & 26.553 & 12.115 & 198 & 592 \\
\hline tech-as-caida2007 & $<0.01$ & $<0.01$ & 746 & 1707 \\
\hline tech-internet-as & 0.026 & 0.022 & 495 & 1409 \\
\hline tech-p2p-gnutella & 0.023 & 0.014 & 746 & 1147 \\
\hline tech-routers-rf & $<0.01$ & $<0.01$ & 914 & 2138 \\
\hline tech-WHOIS & $<0.01$ & $<0.01$ & 940 & 964 \\
\hline scc_enron-only & $<0.01$ & $<0.01$ & 813 & 191 \\
\hline scc_fb-forum & $<0.01$ & $<0.01$ & 719 & 164 \\
\hline scc_fb-messages & $<0.01$ & $<0.01$ & 509 & 75 \\
\hline scc_infect-hyper & $<0.01$ & $<0.01$ & 850 & 163 \\
\hline scc_reality & 0.035 & 0.023 & 323 & 24 \\
\hline scc_retweet & $<0.01$ & $<0.01$ & 914 & 264 \\
\hline scc_retweet-crawl & 0.060 & 0.029 & 513 & 1848 \\
\hline scc_rt_alwefaq & $<0.01$ & $<0.01$ & 842 & 2121 \\
\hline scc_rt_assad & $<0.01$ & $<0.01$ & 849 & 2700 \\
\hline scc_rt_bahrain & $<0.01$ & $<0.01$ & 846 & 4403 \\
\hline scc_rt_barackobama & $<0.01$ & $<0.01$ & 963 & 3600 \\
\hline scc_rt_damascus & $<0.01$ & $<0.01$ & 1105 & 5219 \\
\hline scc_rt_dash & $<0.01$ & $<0.01$ & 780 & 5205 \\
\hline scc_rt_gmanews & $<0.01$ & $<0.01$ & 947 & 1186 \\
\hline scc_rt_gop & $<0.01$ & $<0.01$ & 972 & 7795 \\
\hline scc_rt_http & $<0.01$ & $<0.01$ & 1442 & 7897 \\
\hline scc_rt_israel & $<0.01$ & $<0.01$ & 1107 & 7918 \\
\hline scc_rt_justinbieber & $<0.01$ & $<0.01$ & 1091 & 1452 \\
\hline scc_rt_ksa & $<0.01$ & $<0.01$ & 1058 & 4544 \\
\hline scc_rt_lebanon & $<0.01$ & $<0.01$ & 1338 & 8566 \\
\hline scc_rt_libya & $<0.01$ & $<0.01$ & 901 & 6338 \\
\hline scc_rt_lolgop & $<0.01$ & $<0.01$ & 1156 & 820 \\
\hline scc_rt_mittromney & $<0.01$ & $<0.01$ & 966 & 5342 \\
\hline scc_rt_obama & $<0.01$ & $<0.01$ & 1328 & 9077 \\
\hline scc_rt_occupy & $<0.01$ & $<0.01$ & 777 & 4513 \\
\hline scc_rt_occupywallstnyc & $<0.01$ & $<0.01$ & 907 & 1285 \\
\hline scc_rt_oman & $<0.01$ & $<0.01$ & 801 & 6134 \\
\hline scc_rt_onedirection & $<0.01$ & $<0.01$ & 867 & 864 \\
\hline scc_rt_p2 & $<0.01$ & $<0.01$ & 1019 & 7554 \\
\hline scc_rt_qatif & $<0.01$ & $<0.01$ & 882 & 7185 \\
\hline scc_rt_saudi & $<0.01$ & $<0.01$ & 1004 & 2793 \\
\hline scc_rt_tcot & $<0.01$ & $<0.01$ & 956 & 7273 \\
\hline scc_rt_tlot & $<0.01$ & $<0.01$ & 1010 & 7216 \\
\hline scc_rt_uae & $<0.01$ & $<0.01$ & 946 & 6493 \\
\hline scc_rt_voteonedirection & $<0.01$ & $<0.01$ & 1145 & 7006 \\
\hline scc_twitter-copen & $<0.01$ & 0.01 & 790 & 110 \\
\hline web-edu & $<0.01$ & $<0.01$ & 981 & 2237 \\
\hline web-google & $<0.01$ & $<0.01$ & 754 & 1849 \\
\hline web-indochina-2004 & 0.148 & 0.172 & 629 & 1214 \\
\hline web-polblogs & $<0.01$ & $<0.01$ & 902 & 1942 \\
\hline web-sk-2005 & 15.511 & 6.455 & 424 & 864 \\
\hline web-uk-2005 & 0.045 & 0.038 & 360 & 60 \\
\hline web-webbase- 2001 & 0.024 & $<0.01$ & 695 & 1801 \\
\hline
\end{tabular}


Table 3. Results on the 12 graphs on which NoiseVC makes a substantially large progress

\begin{tabular}{l|l|l||l|l|l}
\hline Graph & $|V|$ & $|E|$ & $\begin{array}{l}\text { FastVC } \times \mathbf{1 0 0} \\
C_{\min }\left(C_{a v g}\right)\end{array}$ & $\begin{array}{l}\text { NoiseVC } \\
C_{\min }\left(C_{a v g}\right)\end{array}$ & $\Delta$ \\
\hline \hline inf-roadNet-CA & 1957027 & 2760388 & $1001272(1001306.3)$ & $\mathbf{1 0 0 1 2 6 9 ( 1 0 0 1 3 0 2 )}$ & 3 \\
inf-roadNet-PA & 1087562 & 1541514 & $555220(555242)$ & $\mathbf{5 5 5 1 9 1}(555226.4)$ & 29 \\
\hline rec-amazon & 91813 & 125704 & $47606(47606)$ & $\mathbf{4 7 6 0 5}(47605.5)$ & 1 \\
\hline sc-pwtk & 217891 & 5653221 & $207712(207717.2)$ & $\mathbf{2 0 7 7 0 7}(207715.2)$ & 5 \\
sc-shipsec1 & 140385 & 1707759 & $117298(117313.8)$ & $\mathbf{1 1 7 2 4 6}(117273.6)$ & 52 \\
sc-shipsec5 & 179104 & 2200076 & $147130(147171.3)$ & $\mathbf{1 4 7 1 1 5}(147142.8)$ & 15 \\
\hline soc-buzznet & 101163 & 2763066 & $30625(30625)$ & $\mathbf{3 0 6 1 8}(30622.3)$ & 7 \\
soc-delicious & 536108 & 1365961 & $85685(85695.5)$ & $\mathbf{8 5 5 2 7}(85596.9)$ & 158 \\
soc-flickr & 513969 & 3190452 & $153272(153272)$ & $\mathbf{1 5 3 2 7 1}(153272)$ & 1 \\
\hline tech-RL-caida & 190914 & 607610 & $74930(74938.9)$ & $\mathbf{7 4 8 6 3}(74883.5)$ & 67 \\
\hline scc_infect-dublin & 10972 & 175573 & $9104(9104)$ & $\mathbf{9 1 0 3 ( 9 1 0 3 )}$ & 1 \\
\hline web-spam & 4767 & 37375 & $2298(2298)$ & $\mathbf{2 2 9 7}(2297)$ & 1 \\
\hline
\end{tabular}

Table 4. Experimental results on different values of $p$ over 10 runs. $\bar{\Delta}$ means the average $\Delta$ on all 136 graphs

\begin{tabular}{c|c|c|c|c|c|c|c|c|c}
\hline$p$ & 0.1 & 0.2 & 0.3 & 0.4 & 0.5 & 0.6 & 0.7 & 0.8 & 0.9 \\
\hline \hline $\bar{\Delta}$ & 5.8 & 4 & 2.7 & 2.2 & 1.2 & 1 & -0.1 & -0.7 & -0.8 \\
\hline
\end{tabular}

\section{Acknowledgment}

This work is supported by ARC Grant FT0991785, NSF Grant No.61463044 and Grant No.[2014]7421 from the Joint Fund of the NSF of Guizhou province of China.

\section{References}

1. Andrade, D.V., Resende, M.G.C., Werneck, R.F.F.: Fast local search for the maximum independent set problem. J. Heuristics 18(4), 525-547 (2012)

2. Barbosa, V.C., Campos, L.C.D.: A novel evolutionary formulation of the maximum independent set problem. J. Comb. Optim. 8(4), 419-437 (2004)

3. Cai, S.: Balance between complexity and quality: local search for minimum vertex cover in massive graphs. In: Proceedings of the Twenty-Fourth International Joint Conference on Artificial Intelligence, IJCAI. pp. 25-31 (2015)

4. Cai, S., Su, K., Chen, Q.: Ewls: A new local search for minimum vertex cover. In: AAAI (2010)

5. Cai, S., Su, K., Luo, C., Sattar, A.: Numvc: An efficient local search algorithm for minimum vertex cover. Journal of Artificial Intelligence Research pp. 687-716 (2013)

6. Cai, S., Su, K., Sattar, A.: Local search with edge weighting and configuration checking heuristics for minimum vertex cover. Artificial Intelligence 175(9), 16721696 (2011)

7. Chung Graham, F., Lu, L.: Complex graphs and networks american mathematical society (2006) 
8. Cormen, T.H., Leiserson, C.E., Rivest, R.L., Stein, C.: Introduction to algorithms, vol. 6. MIT press Cambridge (2001)

9. Eubank, S., Kumar, V., Marathe, M.V., Srinivasan, A., Wang, N.: Structural and algorithmic aspects of massive social networks. In: Proceedings of the fifteenth annual ACM-SIAM symposium on Discrete algorithms. pp. 718-727. Society for Industrial and Applied Mathematics (2004)

10. Ji, Y., Xu, X., Stormo, G.D.: A graph theoretical approach for predicting common RNA secondary structure motifs including pseudoknots in unaligned sequences. Bioinformatics 20(10), 1603-1611 (2004)

11. Jin, Y., Hao, J.: General swap-based multiple neighborhood tabu search for the maximum independent set problem. Eng. Appl. of AI 37, 20-33 (2015)

12. Johnson, D.S., Trick, M.A.: Cliques, coloring, and satisfiability: second DIMACS implementation challenge, vol. 26. American Mathematical Soc., Providence, USA (1996)

13. Katayama, K., Hamamoto, A., Narihisa, H.: An effective local search for the maximum clique problem. Inf. Process. Lett. 95(5), 503-511 (2005)

14. Li, C.M., Quan, Z.: An efficient branch-and-bound algorithm based on maxsat for the maximum clique problem. In: Proc of the Twenty-Fourth AAAI Conference on Artificial Intelligence, AAAI 2010. vol. 10, pp. 128-133. AAAI Press, Atlanta, Georgia, USA (11-15 July 2010)

15. Pullan, W.J., Hoos, H.H.: Dynamic local search for the maximum clique problem. J. Artif. Intell. Res. (JAIR) 25, 159-185 (2006)

16. Richter, S., Helmert, M., Gretton, C.: A stochastic local search approach to vertex cover. In: KI 2007: Advances in Artificial Intelligence, pp. 412-426. Springer (2007)

17. Rossi, R., Ahmed, N.: The network data repository with interactive graph analytics and visualization. In: AAAI. pp. 4292-4293 (2015)

18. Rossi, R.A., Ahmed, N.K.: Coloring large complex networks. Social Network Analysis and Mining 4(1), 1-37 (2014)

19. Rossi, R.A., Gleich, D.F., Gebremedhin, A.H., Patwary, M.M.A.: Fast maximum clique algorithms for large graphs. In: Proceedings of the companion publication of the 23rd international conference on World wide web companion. pp. 365-366. International World Wide Web Conferences Steering Committee (2014)

20. Segundo, P.S., Rodríguez-Losada, D., Jiménez, A.: An exact bit-parallel algorithm for the maximum clique problem. Computers \& OR 38(2), 571-581 (2011)

21. Traud, A.L., Mucha, P.J., Porter, M.A.: Social structure of facebook networks. Physica A: Statistical Mechanics and its Applications 391(16), 4165-4180 (2012)

22. Wu, Q., Hao, J.K.: A review on algorithms for maximum clique problems. European Journal of Operational Research 242(3), 693-709 (2015) 